\title{
INVESTIGACIONES
}

\section{Concepciones alternativas sobre astronomía en estudiantes de educación básica y media de la Región Metropolitana de Chile}

\author{
Alternative conceptions of astronomy in students \\ of basic and high school in the Metropolitan Region of Chile
}

\author{
Felipe Rabanales Loyola ${ }^{a}$ Carlos Vanegas-Ortega ${ }^{b}$ \\ ${ }^{a}$ Universidad Central de Chile. \\ felipe.rabanales@gmail.com \\ ${ }^{b}$ Universidad de Santiago de Chile. \\ cmariov@gmail.com
}

\begin{abstract}
RESUMEN
El aprendizaje de la Astronomía ha presentado diversas dificultades que, en parte, tienen su origen en las concepciones alternativas de los estudiantes. Este estudio buscó identificar las concepciones alternativas recurrentes sobre Astronomía en estudiantes de octavo básico y cuarto año de educación media de la Región Metropolitana de Chile. Para esto, se implementó un test de selección múltiple y grupos focales, con los cuales se identificaron aspectos cuantitativos y cualitativos de las concepciones alternativas. Los resultados evidencian que los estudiantes de octavo básico presentan concepciones alternativas con mayor frecuencia que los de cuarto año de educación media, sin embargo, en el tema de "Estaciones del año", ambos grupos presentaron similar recurrencia, mientras que, al abordar los "Movimientos del Sol en el cielo", se observó que el grupo de cuarto medio presenta concepciones alternativas con más recurrencia que el grupo de octavo básico.
\end{abstract}

Palabras claves: Concepciones alternativas, Astronomía, Enseñanza de la física, Aprendizaje de la física.

\section{ABSTRACT}

The learning of Astronomy has presented various difficulties that, in part, have their origin in the alternative conceptions of the students. This study sought to identify recurring alternative conceptions of Astronomy in eighth grade and fourth year of high school students in the Metropolitan Region of Chile. For this, a multiplechoice test and focus groups has been implemented, with which quantitative and qualitative aspects of alternative conceptions were identified. The results show that students in eighth grade present alternative concepts with more frequently than those in the fourth year of secondary education, however, in the topic of "Seasons of the year", both groups present a similar recurrence, while, when addressing the "Movements of the Sun in the sky", it was observed that the group of fourth year of high school, it presents the alternative conceptions with more recurrence than the group of eighth grade.

Key words: Alternative conceptions, Astronomy, Physics teaching, Learning physics. 


\section{INTRODUCCIÓN}

La Astronomía constituye una disciplina que presenta dificultad en sus aprendizajes debido a varios factores que permiten la existencia de concepciones alternativas en los estudiantes (Zeilik et al., 1998; Vega, 2001; Trumper, 2001; Kriner, 2004; Langhi y Nardi, 2009; Iachel et al., 2008; Gangui et al., 2010; Bretones y Compiani, 2011; Langhi, 2011). La literatura muestra que, indagar y conocer sobre estas concepciones alternativas resulta relevante para lograr aprendizajes significativos y perdurables en los estudiantes, ya que esto le permitiría al docente conocer lo que su estudiantado cree respecto de una noción de Astronomía y, sobre esta base, hacer mejoras adaptativas a sus clases (Campanario y Otero, 2000; Cuellar, 2009; Solbes et al., 2006; Varela et al., 2013; Driver et al., 1999; Martín del Pozo et al., 2013; Driver, 1993). Por ello, el objetivo de esta investigación es determinar cuáles son las concepciones alternativas recurrentes sobre Astronomía de estudiantes de distintos niveles educativos de la Región Metropolitana de Santiago de Chile.

En el contexto de esta investigación se utilizará el término concepciones alternativas para referirse a las representaciones mentales de los estudiantes sobre el mundo natural, que difieren del conocimiento científico actual (Mora y Herrera, 2009). Cubero (1994) plantea que en las últimas décadas se han definido varios términos similares que incluso podrían concebirse como sinónimos de la misma idea que se quiere expresar, por ejemplo, concepciones erróneas, marcos alternativos, ciencia de los niños, razonamiento espontaneo, ideas ingenuas, ideas pre-instruccionales, representaciones y esquemas conceptuales alternativos. En esta investigación se utilizará el término concepciones alternativas para aludir a cualquiera de estos conceptos, cuya definición, mencionada al inicio del párrafo, será común a todas ellas. Además, en este contexto se considera que al incluir palabras como "erróneo" al referirse a estas concepciones es atribuirle un carácter negativo, como también al referirse con el término preconcepto, que alude a algo que no alcanza una cierta categoría, siendo sub-valorado (Cubero, 1994; Martín del Pozo et al., 2013). Las concepciones alternativas no deben menospreciarse ni asociarse con algo perjudicial ya que cumplen un importante rol en el proceso de aprendizaje, y son el punto de partida para que el conocimiento científico sea aprendido por los estudiantes (Cubero, 1994; Martín del Pozo et al., 2013).

Con la teoría del aprendizaje significativo, Ausubel et al. (1983) propuso que, dentro de los factores que más influyen sobre el aprendizaje es lo que el alumno ya conoce de antemano. Esta estructura previa del estudiante, que consiste en ideas, concepciones y conocimientos, se relaciona con la nueva información para así lograr aprendizajes significativos. En este proceso se estimula la motivación del estudiante por lo que se aprende, al tomarle sentido y sentirse implicado en lo estudiado (Martín del Pozo et al., 2013). Bajo esta visión constructivista del aprendizaje de las ciencias, los estudiantes elaboran modelos para representar los fenómenos naturales de su entorno. En esta línea, Driver et al. (1989) señalan que el enfoque del aprendizaje se centra en: a) los conocimientos e ideas del alumno son importantes; b) comprender requiere establecer relaciones de conceptos; c) el que aprende construye significado de forma activa y; d) el alumnado es responsable directo de su propio aprendizaje. De acuerdo con esto, bajo el enfoque constructivista del aprendizaje se debe considerar el rol activo de los estudiantes, mientras que el docente asume un papel facilitador (Driver, 1993).

Siguiendo lo planteado en este enfoque, las concepciones alternativas se pueden considerar como las representaciones mentales que tienen las personas para interpretar 
un proceso o fenómeno, considerándolas como la mejor explicación a estos, sin embargo, difieren del conocimiento científico actual (Mora y Herrera, 2009). Estas concepciones se estructuran por las experiencias personales de cada individuo (ya sean de origen sensorial, cultural o escolar) y están caracterizadas por principios conceptuales, epistemológicos y ontológicos, que forman parte de lo que el alumno ya conoce (Gangui et al., 2010). Cabe señalar, que son más que un almacenaje para consultas posteriores, debido a que representan un filtro conceptual que permite a los alumnos entender el mundo que los rodea (Giordan, 1996).

Ahora bien, considerando lo expuesto por Campanario y Otero (2000), dado que las concepciones alternativas funcionan como marcos conceptuales, también dirigen y orientan el procesamiento de la información que se estudia en los libros o la interpretación de las explicaciones del profesor. Desde el punto de vista constructivista, las concepciones constituyen muchas veces grandes obstáculos para el logro de nuevos aprendizajes, ya que dificultan la adquisición de estos conocimientos al ser persistentes en el esquema mental de los estudiantes (Cubero, 1989; Campanario y Otero, 2000). Por lo anterior, se plantea que las concepciones alternativas representan un factor que debe tenerse en cuenta para planificar la enseñanza y con ello lograr aprendizajes significativos, a su vez, el profesor debe considerar que los alumnos ya poseen un conocimiento científico alternativo, del que tal vez no son conscientes (Driver et al., 1999; Campanario y Otero, 2000; Cuellar, 2009).

Por otra parte, Solbes y Palomar (2011) sostienen que la Astronomía es la rama de la ciencia con más aficionados no profesionales, posiblemente porque trata interrogantes de interés para los seres humanos como el origen del Universo, de dónde provienen los elementos químicos, cuál es el origen de nuestro Sistema Solar, cómo se produjo la vida y cuál será nuestro destino, entre otras. Pese a esto, las carreras científicas que cuentan con un énfasis en matemáticas y física despiertan poco interés en los estudiantes, lo que termina muchas veces en un abandono de las mismas (Solbes et al., 2007; Solbes y Palomar, 2011). Por esto, es importante aprovechar a la Astronomía para despertar el interés en áreas científicas en los estudiantes (Solbes y Palomar, 2011). Lo anterior toma mayor relevancia si se considera que, según las cifras entregadas por el Servicio Nacional de Turismo, para el año 2025 se espera que Chile supere el $70 \%$ de la infraestructura astronómica del planeta.

En el ámbito internacional, hay diversas investigaciones que muestran que el aprendizaje sobre Astronomía presenta serias dificultades (Stahly et al., 1999; Dove, 2002; Trumper, 2001; Trundle et al., 2007). Una de las razones puede ser el alto grado de abstracción de los conceptos y el conocimiento espacial que implica esta disciplina (Kriner, 2004). Además, se ha estudiado que uno de los orígenes de las concepciones alternativas de los alumnos son las concepciones de los propios profesores (Kikas, 2004; Schoon, 1995; Trundle et al., 2002), dentro de las que se destacan las referidas a la explicación de las fases de la Luna, estaciones del año, eclipses, sistema Tierra-Sol-Luna, tamaños y escalas, entre otras (Camino, 1995; Atwood y Atwood, 1995; Atwood y Atwood, 1996; Parker y Heywood, 1998; Vega, 2001; Trumper, 2003; Martínez-Sebastià, 2004; Rutherford, 2004; Pedrochi y Neves, 2005; Ogan-Bekiroglu, 2007; Mulholland y Ginns, 2008; Varela et al., 2015; Huerta, 2017).

En el contexto chileno, son escasos los datos sobre concepciones alternativas en Astronomía. Huerta (2017) indagó sobre ellas en profesores de física en formación, identificando que, dentro de las más frecuentes, están las referidas a los temas de: 
movimientos del Sol en el cielo, fases de la Luna, tamaños y escala del sistema TierraLuna, estaciones del año y gravedad en el espacio. Sin embargo, lo que ocurre con alumnos de nivel escolar sigue siendo una incógnita, que limita las comprensiones sobre los aprendizajes de astronomía de los estudiantes chilenos.

Considerando lo expuesto, el conocer las concepciones alternativas sobre Astronomía de los estudiantes tiene una gran importancia, ya que con esta información los docentes pueden hacer mejores procesos de diseño, aplicación y evaluación de las clases, con el fin de lograr mejores resultados en el aprendizaje de esta área de la ciencia. Por lo tanto, esta investigación busca responder a la siguiente pregunta de investigación: ¿Cuáles son las concepciones alternativas recurrentes sobre Astronomía de estudiantes de distintos niveles educativos de la Región Metropolitana de Santiago de Chile? Cabe señalar que en esta investigación no se definirá hipótesis ya que su alcance es de tipo descriptivo y no busca pronosticar una cifra o un hecho, debido a lo complejo que puede ser realizar una estimación precisa sobre los fenómenos en estudio (Suárez-Iñiguez, 2005; Hernández et al., 2014). No obstante, con el propósito de orientar la investigación se han generado dos preguntas auxiliares:

1. ¿Cuáles son las concepciones alternativas recurrentes sobre Astronomía de estudiantes de octavo básico y cuarto medio de la Región Metropolitana de Santiago de Chile?

2. ¿Qué elementos comunes y diferentes tienen las concepciones alternativas sobre Astronomía entre los estudiantes de octavo básico y cuarto medio de la Región Metropolitana de Santiago de Chile?

Los objetivos específicos de esta investigación son:

$>$ Identificar las concepciones alternativas sobre Astronomía de estudiantes de octavo básico y cuarto medio de la Región Metropolitana de Santiago de Chile.

$>$ Describir los elementos comunes y diferentes que tienen las concepciones alternativas sobre Astronomía entre los estudiantes de octavo básico y cuarto medio de la Región Metropolitana de Santiago de Chile.

\section{METODOLOGÍA}

Esta investigación consiste en un estudio de tipo descriptivo comparativo, con un diseño mixto que incluye aspectos cuantitativos y cualitativos. De esta manera se busca incluir las ventajas de ambos enfoques (Hernández et al., 2014), considerando tanto las respuestas concretas de los estudiantes como las que se pueden compartir en un grupo de conversación sobre temas de Astronomía.

\subsection{POBLACIÓN Y MUESTRA}

De acuerdo con lo establecido por el Ministerio de Educación de Chile (MINEDUC) en sus programas vigentes, se puede identificar la inclusión explicita de los siguientes temas de Astronomía: sistema solar; movimientos de la tierra; fases de la luna; eclipses de luna 
y sol; satélites y asteroides; tamaños; y estructuras cósmicas, los cuales son abordados en los niveles de tercero básico, primero medio y segundo medio (MINEDUC, 2016a; MINEDUC, 2016b).

Considerando lo anterior, esta investigación está enfocada hacia estudiantes de octavo básico y cuarto medio, ya que en estos niveles no se abordan temas de Astronomía, evitando que puedan repetir memorísticamente en sus respuestas lo que hayan estudiado recientemente en la asignatura (Suárez, 2016).

La población corresponde a 71 estudiantes de octavo básico (entre 13 y 14 años de edad) y 41 de cuarto medio (entre 17 y 18 años de edad), pertenecientes a la Región Metropolitana de Chile. Para cumplir con los protocolos éticos, se solicitó tanto el consentimiento de los apoderados como el asentimiento de los estudiantes.

\subsection{INSTRUMENTOS}

Esta investigación cuenta con dos fases de aplicación, en la primera se utilizó un cuestionario de selección múltiple, elaborado con base en un test estandarizado para identificar concepciones alternativas sobre Astronomía (Martín del Pozo et al., 2013). Cabe señalar que se buscó un instrumento pertinente y cercano a los temas de Astronomía especificados por el MINEDUC en sus planes y programas vigentes (MINEDUC, 2013; MINEDUC, 2016a; MINEDUC, 2016b). Con este fin, se seleccionó el Astronomy Diagnostic Test (ADT versión 2.0 en español), desarrollado por Collaboration for Astronomy Education Research (CAER) en 1999 (Hufnagel et al., 2000; Zeilik, 2003) que trata sobre temas como gravitación, tamaños y escalas, fases de la Luna, estaciones del año, movimientos celestiales, Sistema Solar, constelaciones y cielo nocturno. Este test consta de 33 ítems de selección múltiple que presentan entre tres y cinco alternativas, donde se han considerado las primeras 21 preguntas, que están referidas a los temas de Astronomía antes mencionados, cada uno con una opción correcta (que alude al conocimiento científico válido en la actualidad). Dentro de las ventajas que ofrece el trabajar con ítems de este test ya validado para fines similares es que, los distractores representan concepciones alternativas que se han presentado con frecuencia en otros sujetos, lo que también permite realizar comparaciones más específicas con investigaciones previas en cada uno de los temas abordados de Astronomía. Cabe mencionar que se agregaron dos preguntas, en las cuales los estudiantes debían catalogar el nivel de seguridad que sentían en sus respuestas, y el grado de interés que tienen por aprender sobre Astronomía, por lo tanto, el test se compone de 23 ítems en total. Finalmente, se agregó una sección referida a obtener los datos personales de cada estudiante, con el fin de poder identificarlos de acuerdo a sus respuestas, lo que sería necesario para la selección de quiénes participarían en el grupo focal de la segunda fase.

Aunque se trata de un test con validación internacional, debido a las adaptaciones realizadas se llevó a cabo un pilotaje con 26 estudiantes de octavo básico y 24 de cuarto medio de la Región Metropolitana de Chile. De esta manera se pudo reconocer que el tiempo adecuado para su ejecución era de máximo 25 minutos, y para facilitar su comprensión, se cambió la manera de presentar algunos ítems.

En la segunda fase se realizaron dos grupos focales (Hernández et al., 2014; Martín del Pozo et al., 2013), uno para estudiantes de octavo básico y el otro para estudiantes de cuarto medio. Los estudiantes que participaron en esta instancia fueron seleccionados con base en las respuestas del test de selección múltiple, eligiendo a aquellos que: 
- Tuvieron un promedio de aciertos bajo la media. Al tener un promedio de aciertos bajo la media, presentan varias concepciones alternativas, las que podrán ser profundizadas y discutidas en el grupo focal.

- Marcan la opción "interesado(a)" o "muy interesado(a)" al responder el ítem $\mathrm{N}^{\circ} 22$, sobre el grado de interés por aprender sobre Astronomía. Al manifestar su interés hacia la Astronomía, demuestran afinidad hacia los temas que se tratarían en el grupo focal.

- No marcan la opción "nada confiado(a)" al responder el ítem $\mathrm{N}^{\circ} 23$, sobre la seguridad personal en las respuestas entregadas en el test. Al presentar seguridad en sus respuestas, quiere decir que estas no fueron seleccionadas al azar, por lo que tendrían explicaciones o formas de razonamiento que serían elicitables en el grupo focal.

Los grupos focales fueron grabados, con el debido consentimiento de los apoderados de los estudiantes, y en ellos se abordaron 16 preguntas sobre los subtemas de Astronomía tratados en la primera fase. Cada una de estas interrogantes fueron planteadas luego de contextualizar en cada caso una situación específica, a veces con ayuda de videos o fotografías, para indagar sobre la opinión en general de los estudiantes, registrando la narrativa colectiva que se fue construyendo entre ellos (Hernández et al., 2014). El instrumento utilizado en esta segunda fase fue revisado y validado por investigadores en didácticas de la física, lo que permitió garantizar la validez y confiabilidad de la información recogida (Cabero y Llorente, 2013).

\subsection{PROCESAMIENTO DE LA INFORMACIÓN}

Los datos obtenidos en el test fueron sistematizados en tablas de Excel y clasificados temáticamente (Braun y Clarke, 2006), de acuerdo a las dimensiones correspondientes. De esta manera se pudo determinar la frecuencia que tuvo cada alternativa de los ítems, y con esto, qué concepciones alternativas se presentan de manera más recurrente en cada grupo de estudiantes (Gangui et al., 2007). Con esta información, se ajustaron algunas preguntas del grupo focal, para así poder indagar más sobre las concepciones alternativas que habían sido identificadas en el test. La información obtenida de los grupos focales permitió identificar las principales ideas fuerza que se construyeron con la opinión colectiva, analizando si esta información se condice con los datos cuantitativos individuales.

\section{RESULTADOS Y ANÁLISIS}

A continuación, se presentan los resultados obtenidos para cada grupo de estudiantes (octavo básico y cuarto medio) de acuerdo a las distintas dimensiones que se abordaron tanto en los test y como en los grupos focales correspondientes.

Los resultados generales sobre las preguntas de "Gravedad" se sintetizan en la Tabla 1. 
Estudios Pedagógicos XLVII N 2: 247-268, 2021 CONCEPCIONES ALTERNATIVAS SOBRE ASTRONOMÍA EN ESTUDIANTES DE EDUCACIÓN BÁSICA Y MEDIA DE LA REGIÓN METROPOLITANA DE CHILE

Tabla 1. Porcentaje de respuestas correctas e incorrectas en el test, sobre Gravedad

\begin{tabular}{|c|c|c|c|c|}
\hline \multirow{2}{*}{$\begin{array}{c}\text { Dimensión } \\
\left(\mathrm{N}^{\circ} \text { pregunta del test }\right)\end{array}$} & \multicolumn{2}{|c|}{ Octavo básico } & \multicolumn{2}{c|}{ Cuarto medio } \\
\cline { 2 - 5 } & Incorrectas & Correctas & Incorrectas & Correctas \\
\hline Gravedad $(1-3)$ & $78,4 \%$ & $21,6 \%$ & $52,8 \%$ & $47,2 \%$ \\
\hline
\end{tabular}

En alumnos de octavo básico se puede reconocer la recurrencia de concepciones alternativas como: los objetos más pesados caen más rápido que los livianos $(60,6 \%)$, la presencia de la atmósfera hace que los cuerpos pesen más $(47,9 \%)$ y, cuando son consultados sobre por qué los astronautas del Transbordador espacial flotan en la cabina, solo el 1,4\% acertó en su respuesta, mientras que la mayoría de las preferencias se las llevó la opción de que no hay gravedad en el espacio (64,8 \%). Esto último se condice con lo expuesto por los estudiantes en el grupo focal, según se puede apreciar en el siguiente fragmento discursivo:

Investigador: ¿Por qué creen que los objetos en órbita, incluso los líquidos, pueden flotar, a diferencia de lo que ocurre en donde nos encontramos nosotros?

Estudiante 1: Porque allá hay "gravedad cero"

Investigador: ¿Alguien más está de acuerdo con lo que plantea el Estudiante 1? *Cuatro estudiantes más levantan la mano en señal de aprobación.

Investigador: ¿Alguien que no esté de acuerdo con la respuesta del Estudiante 1? *Todos manifiestan estar de acuerdo con lo planteado por el Estudiante 1.

Investigador: Pero ¿existirá la posibilidad de que haya gravedad ahí?

Estudiante 2: $\quad$ Es que en la Tierra todo cae hacia abajo, todo se empuja hacia abajo, en cambio en el espacio los objetos se van hacia todas partes, no solamente hacia abajo.

Investigador: ¿Pero hay gravedad?

Estudiante 2: $\quad$ Mmmm, no sé.

Estudiante 3: Yo creo que debe haber gravedad, ya que está orbitando. No es como que esté fuera de la órbita, ya que, si no, se iría, pero como está orbitando, hay gravedad.

Investigador: ¿Y por qué el objeto no cae hacia abajo?

Estudiante 3: $\quad$ Porque es mucho menor la gravedad, bastante menor.

Por su parte, en alumnos de cuarto medio la concepción alternativa que tuvo una mayor frecuencia que la opción correcta es que no hay gravedad en el espacio (51,2 \%) y, al igual que en el otro grupo, existe un bajo el nivel de acierto en esta pregunta $(9,8 \%)$. También, la concepción alternativa que atribuye a la atmósfera la responsabilidad que uno pese más, tiene una considerable recurrencia del 34,1\%. En cuanto a lo expuesto en el grupo focal, los estudiantes de cuarto medio plantean que existe muy poca gravedad en el espacio alrededor de la Tierra, lo que permite que los objetos en órbita puedan flotar allí, sin caer a la superficie. 
Cabe señalar que en las preguntas introductorias del grupo focal se les planteó lo siguiente: si tuvieran la oportunidad, ¿qué le preguntarían a un astronauta? En ambos grupos surgieron las ideas de consultarles “¿qué se siente estar en el espacio donde no hay gravedad?", lo que se condice con sus respuestas del test y del grupo focal.

Para un análisis más detallado, se presenta a continuación los resultados del ítem $\mathrm{N}^{\circ}$ 2 del test:

Los astronautas dentro del Transbordador Espacial flotan en la cabina al orbitar la Tierra porque:
A) No hay gravedad en el espacio.
B) Están cayendo de la misma manera que el Trasbordador Espacial.
C) Se encuentran sobre la atmósfera terrestre.
D) Se encuentran en una menor gravedad dentro del Transbordador Espacial.

Figura 1. Porcentaje de respuestas de los estudiantes de octavo básico y cuarto medio para el ítem $\mathrm{N}^{\circ} 2$ del test, perteneciente a la dimensión Gravedad.

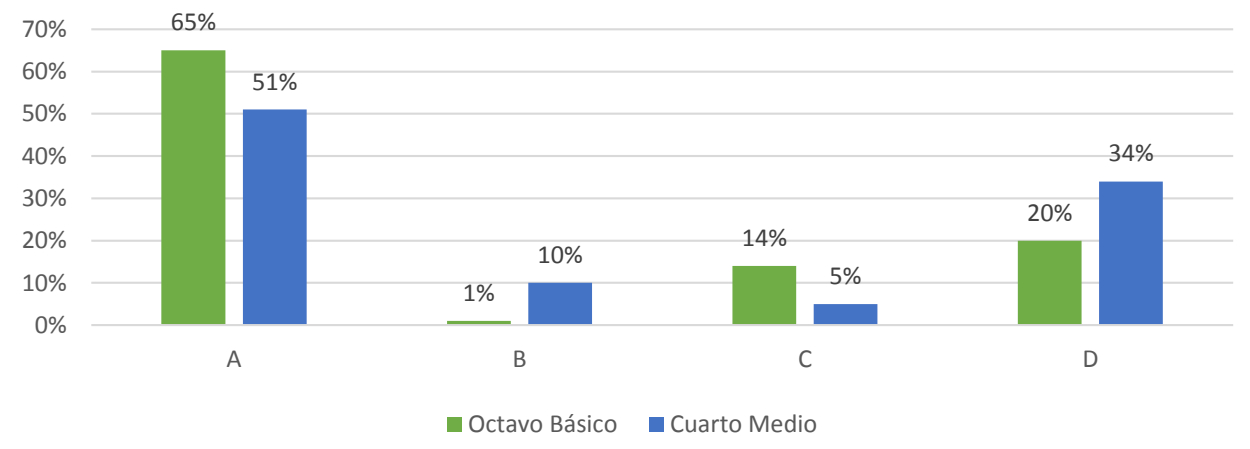

Las respuestas obtenidas en el ítem $\mathrm{N}^{\circ} 2$ dejan en evidencia que la concepción alternativa asociada a que en el espacio no hay gravedad es muy recurrente en ambos grupos (65\% para octavo básico y $51 \%$ para cuarto medio), mientras que el porcentaje de acierto en la opción correcta (B) tiene baja frecuencia (1 \% y $10 \%$ respectivamente). Los mejores resultados obtenidos por el grupo de cuarto medio pueden deberse a la formación sobre temas de gravitación que han tenido en su enseñanza escolar, a diferencia de los alumnos de octavo básico. Este mismo hecho puede haber influido también en que ellos no consideran mayormente el efecto que pueda provocar la atmósfera en la gravedad (5\%, opción C), sin embargo, la opción D presenta una considerable frecuencia (34 \%, incluso mayor que el $20 \%$ del grupo de octavo básico), con una concepción alternativa que debe tenerse en cuenta para los procesos de enseñanza.

De investigaciones previas se pueden destacar los resultados obtenidos por Gangui et al. (2010) que, en un estudio con profesores en formación, el $17 \%$ no responde correctamente sobre temas básicos de gravedad y caída de los cuerpos. Similares resultados se aprecian 
en los estudios de Varela et al. (2015), donde solo el $16 \%$ de los profesores en formación, responde correctamente sobre la fuerza gravitatoria entre la Luna y la Tierra, mientras que en el estudio realizado por Rutherford (2004) con profesores, este porcentaje se mantiene en un $18 \%$, identificando incluso a un $29 \%$ que sostuvo que la Luna no produce gravedad.

Los resultados generales sobre las preguntas de "Dimensiones y Escalas" se sintetizan en la Tabla 2.

Tabla 2. Porcentaje de respuestas correctas e incorrectas en el test, sobre Dimensiones y Escalas

\begin{tabular}{|c|c|c|c|c|}
\hline \multirow{2}{*}{$\begin{array}{c}\text { Dimensión } \\
\left(\mathrm{N}^{\circ} \text { pregunta del test }\right)\end{array}$} & \multicolumn{2}{|c|}{ Octavo básico } & \multicolumn{2}{c|}{ Cuarto medio } \\
\cline { 2 - 5 } & Incorrectas & Correctas & Incorrectas & Correctas \\
\hline Dimensiones y Escalas $(4-7)$ & $69,4 \%$ & $30,6 \%$ & $51,8 \%$ & $48,2 \%$ \\
\hline
\end{tabular}

En estudiantes de octavo básico se identificaron concepciones alternativas como: la distancia entre la Tierra y la Luna se estima como máximo con un $10 \%$ del valor real $(67,7 \%)$, y también, que la Estación espacial internacional se encuentra aproximadamente a medio camino entre la Tierra y la Luna (46,5\%). En el grupo focal se pudo reconocer que, en general, consideran que el diámetro de la Luna es del orden del $50 \%$ del que tiene la Tierra (siendo aproximadamente $27,3 \%$ el valor real), mientras que, sobre la distancia Tierra-Luna, no hay un consenso claro ya que algunos la asumen muy pequeña y otros muy grande con relación a su dimensión real. Respecto de la distancia entre la Tierra y Marte, en general, los estudiantes de octavo básico consideran que tiene una dimensión aproximada de entre veinte y cuarenta veces menor que el valor real.

Por otro lado, en alumnos de cuarto medio las concepciones alternativas que se pueden reconocer son similares a las del grupo anterior, con distinta frecuencia: la distancia Tierra-Luna se estima como máximo con un $10 \%$ del valor real $(48,8 \%)$, y, un 39,0 \% de los estudiantes considera que la Estación espacial internacional está ubicada a medio camino entre la Tierra y la Luna. En el grupo focal se pudo identificar que los estudiantes consideran que el diámetro de la Luna es aproximadamente el $22 \%$ del que tiene la Tierra, mientras que la distancia Tierra-Luna, en general, se cree que es muy pequeña con relación al valor real. Finalmente, respecto de la distancia Tierra-Marte, se consideró que tiene una dimensión de entre doscientas y dos mil veces menor que el valor real.

En las Figuras 2 y 3 se presentan algunos de los dibujos realizados por estudiantes de octavo básico y cuarto medio, cuando se les pidió representar el tamaño a escala de la Tierra y la Luna, junto con la separación que hay entre ambas. 
Figura 2. Dibujos realizados por dos estudiantes de octavo básico, sobre el tamaño de la Tierra (T) y la Luna (L), junto con la distancia que las separa.

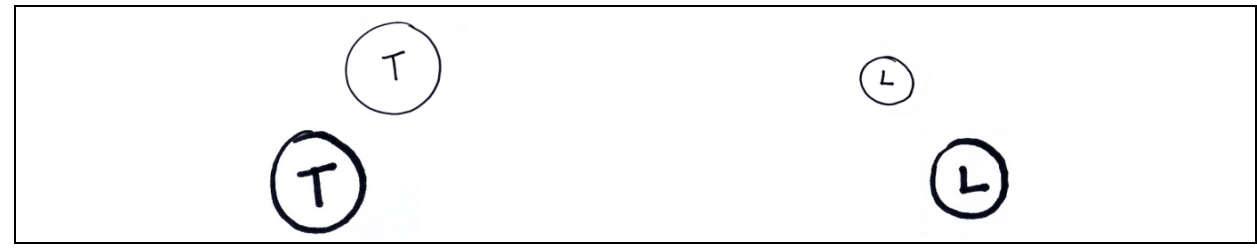

Figura 3. Dibujos realizados por tres estudiantes de cuarto medio, sobre el tamaño de la Tierra (T) y la Luna (L), junto con la distancia que las separa.

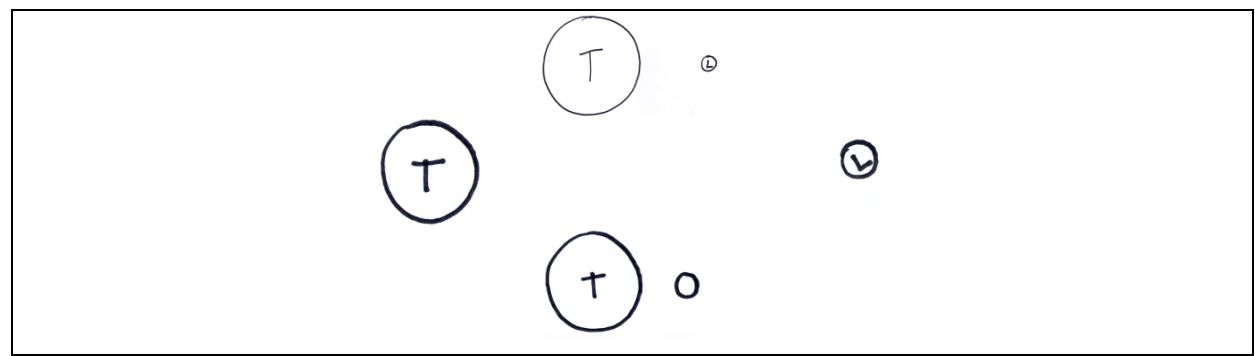

Los resultados generales sobre las preguntas de "Fases de la Luna" se sintetizan en la Tabla 3.

Tabla 3. Porcentaje de respuestas correctas e incorrectas en el test, sobre Fases de la Luna

\begin{tabular}{|c|c|c|c|c|}
\hline \multirow{2}{*}{$\begin{array}{c}\text { Dimensión } \\
\left(\mathrm{N}^{\circ} \text { pregunta del test }\right)\end{array}$} & \multicolumn{2}{|c|}{ Octavo básico } & \multicolumn{2}{c|}{ Cuarto medio } \\
\cline { 2 - 5 } & Incorrectas & Correctas & Incorrectas & Correctas \\
\hline Fases de la Luna $(8-10)$ & $79,2 \%$ & $20,8 \%$ & $63,3 \%$ & $36,7 \%$ \\
\hline
\end{tabular}

En el grupo de octavo básico se presentaron concepciones alternativas recurrentes de que la Luna varía notoriamente su luminosidad durante una misma noche $(71,8 \%)$, como también que, en un eclipse solar la Luna se encuentra en fase llena $(75,4 \%)$. Esta última concepción no se presentó al momento de realizar el grupo focal, sin embargo, hay que considerar que en el país había ocurrido hace algunos días un eclipse solar, que involucró mucha información al respecto en todos los medios de comunicación. Pese a tener claro las fases de la Luna que ocurren en los eclipses lunares y solares, demuestran no estar seguros de la posición que ocupan la Tierra, el Sol y la Luna en cada caso.

En cuanto a los alumnos de cuarto medio, se presentan las mismas concepciones alternativas anteriores, la rápida variación de la luminosidad de la Luna en la noche con un 
$53,7 \%$, mientras que la presencia de Luna llena cuando hay eclipse solar tuvo una frecuencia del $65,0 \%$. En el grupo focal se logró identificar también la concepción alternativa relativa a que, la sombra de la Tierra proyectada en la Luna influye en que se produzcan las fases lunares. Al igual que en el grupo de octavo, los estudiantes reconocen correctamente en qué fase está la Luna en cada eclipse, y en este caso, identifican correctamente la posición que tienen la Tierra, la Luna y el Sol en cada caso.

En estudios sobre este tema, autores como Camino (1995), Stahly et al. (1999), Parker y Heywood (1998) y Trumper (2001) reconocen que la principal explicación de los estudiantes al fenómeno de fases de la Luna es lo que ocurre cuando ésta se sitúa en la sombra que produce la Tierra, y en menor medida, opciones como la influencia de la sombra que producen otros planetas y nubes que la puedan cubrir. Iachel et al. (2008) identifican en alumnos de educación secundaria que solo el $20 \%$ explica correctamente las fases de la Luna, el 42,5 \% no sabe a qué se debe, y el 17,5 \% lo confunde con el fenómeno de eclipse. Por su parte, Vega (2001) en su estudio con profesores encontró que el $63 \%$ no explica correctamente dónde está la Luna durante el día, además, que el 64 \% considera que la Luna no se mueve. Esto último también ha sido reconocido por Gangui et al. (2010) con profesores en formación, donde solo el $12 \%$ reconoce que la Luna tiene movimientos de rotación y traslación. Los resultados de todos estos estudios son complementados con las concepciones alternativas recurrentes que se identificaron en esta investigación, demostrando que existen diversas concepciones en temas relativos a las fases de la Luna, sus movimientos, e incluso los eclipses. Finalmente, Varela et al. (2015), en su estudio con profesores, reconoce que el $69 \%$ responde erróneamente sobre qué causa las fases de la Luna, mientras que un $78 \%$ asocia el eclipse de Sol con la Luna llena, resultados que son similares a los porcentajes obtenidos tanto en esta investigación como los de Trumper (2001).

Los resultados generales sobre las preguntas de "Estaciones del año" se sintetizan en la Tabla 4.

Tabla 4. Porcentaje de respuestas correctas e incorrectas en el test, sobre Estaciones del año

\begin{tabular}{|c|c|c|c|c|}
\hline \multirow{2}{*}{$\begin{array}{c}\text { Dimensión } \\
\left(\mathrm{N}^{\circ} \text { pregunta del test }\right)\end{array}$} & \multicolumn{2}{|c|}{ Octavo básico } & \multicolumn{2}{c|}{ Cuarto medio } \\
\cline { 2 - 5 } & Incorrectas & Correctas & Incorrectas & Correctas \\
\hline Estaciones del año $(11)$ & $88,7 \%$ & $11,3 \%$ & $87,8 \%$ & $12,2 \%$ \\
\hline
\end{tabular}

En los estudiantes de octavo básico se identificó que la concepción alternativa recurrente es que las estaciones del año (otoño, invierno, primavera y verano) se producen exclusivamente por la trayectoria elíptica que tiene la Tierra entorno al Sol $(39,4 \%)$, y que éstas no se producirían si la trayectoria de la Tierra fuera perfectamente circunferencial. En el grupo focal se ratifica esta concepción, ya que la mayoría atribuye a la cercanía o lejanía de la Tierra respecto del Sol como la causa de que se provoquen las estaciones del año, sin embargo, al ser informados sobre las distintas estaciones que se provocan en diferentes ciudades del mundo en un mismo momento, ponen en duda sus posturas iniciales, considerando la opción de que podría influir la manera en que llega la radiación solar a las ciudades, como el factor que determine las estaciones del año. 
Por otro lado, en estudiantes de cuarto medio, esta misma concepción alternativa se afianza aún más, con un 46,3\%. En el grupo focal se pudo constatar que, tal como en el caso anterior, al ser consultados sobre por qué se provocan las estaciones del año, la mayoría tiende a responsabilizar a la distancia entre el Sol y la Tierra, sin embargo, cuando se les indica que, distintas estaciones ocurren al mismo tiempo en diferentes partes del planeta, notan que esta explicación no es satisfactoria, y se convencen de que la inclinación del eje terrestre es la causante. Esta explicación había sido planteada inicialmente por uno de los integrantes del grupo.

Para ilustrar estas concepciones recurrentes, a continuación, se presentan los resultados del ítem $\mathrm{N}^{\circ} 11$ del test:

Imagina que la órbita de la Tierra fuera cambiada a ser una circunferencia perfecta alrededor del Sol, tal que la distancia al Sol nunca cambiaría. ¿Cómo afectaría esto a las estaciones del año?

A) Ya no experimentaríamos diferencias entre las estaciones del año.

B) Continuaríamos experimentando las estaciones, pero la diferencia entre ellas sería mucho MENOS notoria.

C) Continuaríamos experimentando las estaciones, pero la diferencia entre ellas sería mucho MÁS notoria.

D) Continuaríamos experimentando las estaciones del año de la misma manera que ahora.

Figura 4. Porcentaje de respuestas de los estudiantes de octavo básico y cuarto medio para el ítem $\mathrm{N}^{\mathrm{o}} 11$ del test, perteneciente a la dimensión Estaciones del año.

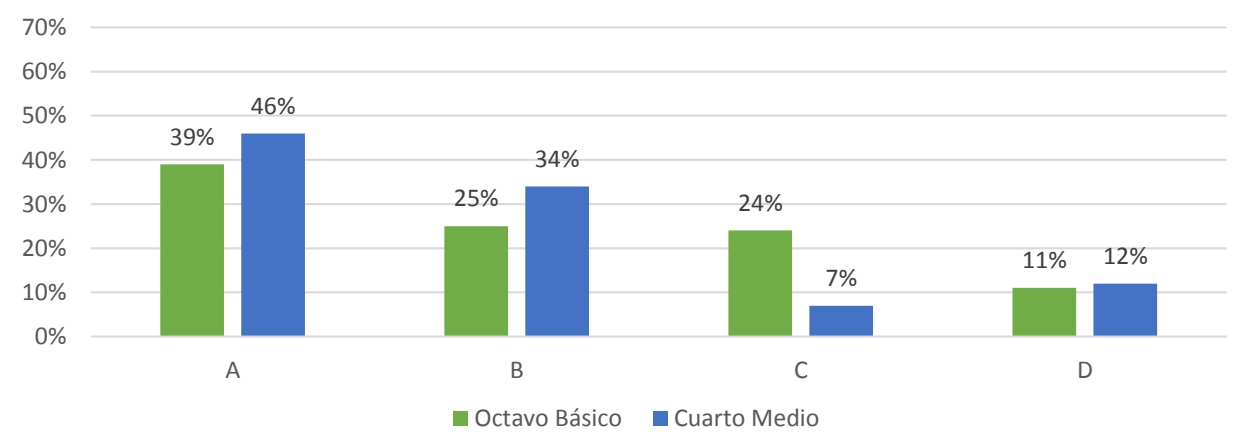

Como se puede apreciar en estos resultados sobre el fenómeno de las estaciones del año, la concepción alternativa más recurrente es responsabilizar a la forma no perfectamente circunferencial de la órbita terrestre en torno al Sol (39 \% en octavo básico y un $46 \%$ en cuarto medio). El mayor porcentaje presente en alumnos de cuarto medio evidencia que esta concepción tiende a mantenerse con el paso de los años e incluso puede arraigarse aún más en estudiantes de enseñanza media que en los de la básica. Por su parte, la frecuencia 
que presenta la opción correcta (D) es prácticamente la misma en ambos grupos (entre 11 $\%$ y $12 \%$ ), por tanto, la mayoría de los estudiantes considera que se esperaría un efecto notorio en las estaciones del año si la órbita de la Tierra fuera perfectamente circunferencial alrededor del Sol, lo cual, puede deberse a que consideren que la órbita actual tiene una gran excentricidad.

Lo anterior se complementa con estudios previos como los de Camino (1995) y Trumper (2001), con profesores, Pedrochi y Neves (2005) y Rutherford (2004), con profesores en formación, para quienes la principal explicación que le atribuye al fenómeno de estaciones del año es la variación de la distancia entre la Tierra y el Sol. En investigaciones anteriores se han detectado variados porcentajes de preferencia a esta concepción, por ejemplo, con profesores en formación, Martínez-Sebastiá (2004) determinó un $81 \%$ y Gangui et al. (2010) un $41 \%$, mientras que, con profesores, Varela et al. (2015) pudo identificar un 29 $\%$ de recurrencia. Lo anterior, puede deberse en parte a la forma de la órbita que se cree que describe la Tierra alrededor del Sol, tomando en cuenta que en el estudio realizado por Varela et al. (2015) solo un $6 \%$ de los profesores responde correctamente sobre este tema, siendo un $63 \%$ los que se inclinan hacia la opción que la órbita terrestre es elíptica con una gran excentricidad, y el Sol en uno de sus focos.

Los resultados generales sobre las preguntas de "Movimientos del Sol en el cielo" se sintetizan en la Tabla 5.

Tabla 5. Porcentaje de respuestas correctas e incorrectas en el test, sobre Movimientos del Sol en el cielo

\begin{tabular}{|c|c|c|c|c|}
\hline \multirow{2}{*}{$\begin{array}{c}\text { Dimensión } \\
\left(\mathrm{N}^{\circ} \text { pregunta del test }\right)\end{array}$} & \multicolumn{2}{|c|}{ Octavo básico } & \multicolumn{2}{c|}{ Cuarto medio } \\
\cline { 2 - 5 } & Incorrectas & Correctas & Incorrectas & Correctas \\
\hline Movimiento del Sol en el cielo $(12-13)$ & $73,3 \%$ & $26,7 \%$ & $81,7 \%$ & $18,3 \%$ \\
\hline
\end{tabular}

En el grupo de octavo básico se pudo reconocer que, un 77,5\% considera que en la ciudad donde ellos viven, Santiago de Chile, el Sol se encuentra en el cenit a lo menos un día del año, incluso, un 42,3 \% considera que dicho fenómeno ocurre todos los días al mediodía. Esto último se contrapone a lo observado en el grupo focal, ya que se expuso que, solo en países donde pase la línea del Ecuador podría observarse al Sol en el cenit, o a lo menos, en regiones que se encuentran cercanas a este punto de referencia.

Por su parte, en los estudiantes de cuarto medio se presentan las mismas concepciones alternativas que el grupo anterior, pero con mayor porcentaje en ambos casos: un 85,4 \% considera que, desde Santiago de Chile, al menos un día del año el Sol se ubica en el cenit, siendo esta vez un 51,2 \% los que consideran que esto ocurre todos los días al mediodía. Lo anterior se complementa con el siguiente fragmento discursivo, que corresponde a lo observado en el grupo focal:

Para un análisis más detallado, se presenta a continuación el ítem $\mathrm{N}^{\circ} 12$ del test: 
Considerando lo que percibimos en Santiago, ¿cuándo es que el asta de bandera vertical no produce sombra alguna debido a que el Sol se encuentra directamente sobre el asta?
A) Todos los días al mediodía.
B) Solamente el primer día del verano.
C) Solamente el primer día del invierno.
D) El primer día de la primavera y el primer día del otoño.
E) Nunca desde esta ciudad.

Figura 5. Porcentaje de respuestas de los estudiantes de octavo básico y cuarto medio para el ítem $\mathrm{N}^{\mathrm{o}} 12$ del test, perteneciente a la dimensión Movimientos del Sol en el cielo.

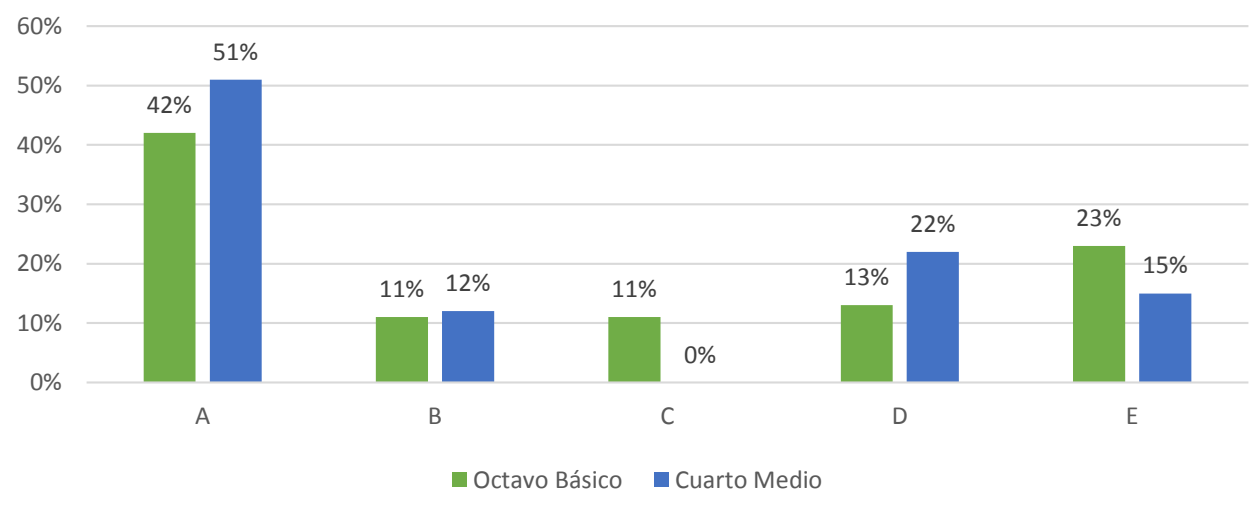

Los resultados obtenidos muestran que la concepción alternativa más recurrente es que el Sol se puede observar en el cenit todos los días del año desde la ciudad en que se realizó este estudio (Santiago de Chile), teniendo una frecuencia mayor en el grupo de cuarto medio $(51 \%)$ que en el grupo de octavo básico (42\%). A esto se suma que la opción correcta (E) tuvo mayores preferencias en el grupo de octavo básico (23\%) que en cuarto medio (15\%), lo cual podría estar ocurriendo porque este tema no se aborda directamente en la enseñanza escolar chilena, permitiendo un mayor asentamiento de ciertas concepciones alternativas. Cabe destacar que los resultados de este ítem indican que los estudiantes, en general, no se dedican a observan con detención fenómenos astronómicos cotidianos como la trayectoria del Sol durante los días, ya que al menos la opción A se podría refutar con reconocer un solo caso que lo contradiga (esta investigación se realizó al inicio del invierno, donde es más fácil notar que este fenómeno no ocurre). Por otro lado, la opción D, sobre todo para el grupo de cuarto medio (22\%), indica que el fenómeno en cuestión se suele asociar a los equinoccios.

Lo anterior se complementa con lo encontrado por Varela et al. (2015), con profesores, al determinar que sobre el $90 \%$ considera que, desde su punto de vista, el Sol se podría observar en el cenit (en una ciudad que, al igual que en Santiago de Chile, no es posible que ocurra por su latitud), siendo incluso un $41 \%$ los que consideran que esto ocurre todos los días, similar a lo encontrado por Trumper (2001) con estudiantes escolares y Rutherford (2004) en profesores. Martínez-Sebastiá (2004), con profesores en formación, identifica 
otros aspectos relevantes a este tema: el $86 \%$ considera que el lugar por donde sale el Sol no varía a lo largo del año; un $58 \%$ considera que no cambia la altura máxima que alcanza el Sol en el cielo; solo el $23 \%$ son capaces de reconocer los equinoccios y un $9 \%$ los solsticios; además que, ningún estudiante fue capaz de justificar por qué se provocan los cambios en la posición de salida del Sol en distintos períodos del año.

Los resultados generales sobre las preguntas de "Constelaciones" se sintetizan en la Tabla 6.

Tabla 6. Porcentaje de respuestas correctas e incorrectas en el test, sobre Constelaciones

\begin{tabular}{|c|c|c|c|c|}
\hline \multirow{2}{*}{$\begin{array}{c}\text { Dimensión } \\
\left(\mathrm{N}^{\circ} \text { pregunta del test }\right)\end{array}$} & \multicolumn{2}{|c|}{ Octavo básico } & \multicolumn{2}{c|}{ Cuarto medio } \\
\cline { 2 - 5 } & Incorrectas & Correctas & Incorrectas & Correctas \\
\hline Constelaciones $(14-15)$ & $80,3 \%$ & $19,6 \%$ & $61,0 \%$ & $39,0 \%$ \\
\hline
\end{tabular}

En alumnos de octavo básico la concepción alternativa recurrente que se pudo identificar es que el Sol varía notoriamente su posición, con relación a las demás estrellas del cielo, durante un día, esto nos permitiría ver al Sol pasando de una constelación a otra con el paso de las horas $(84,5 \%)$. Además, un 76,1 \% manifiesta que, podríamos observar un cambio importante en la figura de una constelación, como la conocemos desde nuestro planeta ( nuestro Sistema Solar. Esto último se condice con lo expuesto en el grupo focal, donde los estudiantes consideraban que este cambio en una constelación se podría notar solo con el hecho de viajar a otro punto del planeta, o también con la traslación de la Tierra en torno al Sol.

En cuanto a lo que ocurre en el grupo de cuarto medio, en el 70,7 \% también se presenta la concepción alternativa relativa a que el Sol recorre distintas constelaciones con el paso de las horas en un día. En tanto, un 51,2 \% afirma que, situándonos en algún otro punto dentro de nuestro Sistema Solar nos permitiría observar un cambio considerable en la forma de las constelaciones como las conocemos. En el grupo focal se pudo apreciar más notoriamente esta última concepción alternativa, ya que se consideraba que un viaje dentro del Sistema Solar permitiría percibir las constelaciones con otra forma y/o tamaño.

En cuanto a las preguntas sobre "Estrellas", en la Tabla 7 se presentan los resultados generales.

Tabla 7. Porcentaje de respuestas correctas e incorrectas en el test, sobre Estrellas

\begin{tabular}{|c|c|c|c|c|}
\hline \multirow{2}{*}{$\begin{array}{c}\text { Dimensión } \\
\left(\mathrm{N}^{\circ} \text { pregunta del test }\right)\end{array}$} & \multicolumn{2}{|c|}{ Octavo básico } & \multicolumn{2}{c|}{ Cuarto medio } \\
\cline { 2 - 5 } & Incorrectas & Correctas & Incorrectas & Correctas \\
\hline Estrellas $(16-18)$ & $80,6 \%$ & $19,4 \%$ & $57,7 \%$ & $42,3 \%$ \\
\hline
\end{tabular}


En estudiantes de octavo básico las concepciones alternativas que se presentaron fueron: las estrellas más calientes tienen un color cálido, como el rojo o naranjo $(52,1$ $\%)$, la energía del Sol proviene del calor remanente del Big Bang $(34,3 \%)$ y, por otro lado, la luminosidad de una fuente que emite luz disminuye de manera inversamente proporcional con la distancia a ella $(72,9 \%)$. Por otro lado, en el grupo focal se pudo identificar la concepción de que las estrellas son más brillantes al ser más jóvenes, y su brillo va disminuyendo con el pasar del tiempo.

En tanto, en el grupo de cuarto medio las concepciones alternativas recurrentes fueron similares: un 43,9\% referida a que las estrellas más calientes son las que tienen color rojo o naranjo (con mucha más frecuencia el rojo), y también que, la luminosidad de una fuente de luz disminuye inversamente proporcional con la distancia $(56,1 \%)$. Por su parte, en el grupo focal fue recurrente la concepción de que las estrellas más brillantes del cielo están más cerca que las que tienen una menor luminosidad.

Los resultados generales sobre las preguntas de "Otros temas de Astronomía" se sintetizan en la Tabla 8 .

Tabla 8. Porcentaje de respuestas correctas e incorrectas en el test, sobre Otros temas de Astronomía

\begin{tabular}{|c|c|c|c|c|}
\hline \multirow{2}{*}{$\begin{array}{c}\text { Dimensión } \\
\left(\mathrm{N}^{\circ} \text { pregunta del test }\right)\end{array}$} & \multicolumn{2}{|c|}{ Octavo básico } & \multicolumn{2}{c|}{ Cuarto medio } \\
\cline { 2 - 5 } & Incorrectas & Correctas & Incorrectas & Correctas \\
\hline Otros $(19-21)$ & $67,1 \%$ & $32,8 \%$ & $48,0 \%$ & $52,0 \%$ \\
\hline
\end{tabular}

En el grupo de octavo básico las concepciones alternativas recurrentes fueron: las ondas de radio se propagan mucho más lentamente que la luz visible $(73,2 \%)$, y, el calentamiento global es causado mayoritariamente por la destrucción de la capa de Ozono (54,9\%).

Por otro lado, en el grupo de cuarto medio también se presentó la concepción alternativa relativa a que las ondas de radio viajan mucho más lentas que la luz visible $(51,2 \%)$ sin embargo, hay menos frecuencia que en el grupo de octavo básico, esto podría ocurrir porque es un contenido revisado en la enseñanza escolar, de la misma manera que lo referido al calentamiento global.

\section{CONCLUSIONES}

Los resultados obtenidos en este estudio han coincidido con los encontrados en algunas investigaciones previas, como las de Gangui et al. (2010); Varela et al. (2015); Rutherford (2004); Camino (1995); Stahly et al. (1999); Parker y Heywood (1998); Trumper (2001); Iachel et al. (2008); Gangui et al. (2010); Pedrochi y Neves (2005); Martínez-Sebastiá (2004) y Huerta (2017). En las tablas 9 y 10 se presenta un resumen de las principales concepciones alternativas identificadas para cada grupo de estudiantes, con su respectivo porcentaje de recurrencia en las respuestas. 
Tabla 9. Concepciones alternativas recurrentes en estudiantes de octavo básico

\begin{tabular}{|c|c|c|}
\hline $\mathrm{N}^{\circ}$ & Concepción alternativa & $\%$ de respuestas \\
\hline $1 \mathrm{a}$ & En el espacio no hay gravedad & 64,8 \\
\hline $2 \mathrm{a}$ & La aceleración de gravedad es mayor en objetos más pesados & 60,6 \\
\hline $3 \mathrm{a}$ & La atmósfera aumenta el peso de los objetos & 47,9 \\
\hline $4 \mathrm{a}$ & $\begin{array}{l}\text { La distancia Tierra-Luna es estimada como máximo en un } 10 \% \text { del } \\
\text { valor real }\end{array}$ & 67,7 \\
\hline $5 \mathrm{a}$ & $\begin{array}{l}\text { La Estación espacial internacional se ubica aproximadamente a } \\
\text { media distancia entre la Tierra y la Luna }\end{array}$ & 46,5 \\
\hline $6 \mathrm{a}$ & $\begin{array}{l}\text { La luminosidad de la Luna varía notoriamente durante una misma } \\
\text { noche }\end{array}$ & 71,8 \\
\hline $7 \mathrm{a}$ & La luna se encuentra en fase llena cuando ocurre un eclipse solar & 75,4 \\
\hline $8 \mathrm{a}$ & $\begin{array}{l}\text { Las estaciones del año se producen exclusivamente por la variación } \\
\text { de la distancia Tierra-Sol }\end{array}$ & 39,4 \\
\hline $9 \mathrm{a}$ & El Sol se ubica en el cenit a lo menos un día en el año & 77,5 \\
\hline $10 \mathrm{a}$ & El Sol se ubica en el cenit todos los días del año al mediodía & 42,3 \\
\hline $11 \mathrm{a}$ & El Sol recorre distintas constelaciones en el cielo a lo largo de un día & 84,5 \\
\hline $12 \mathrm{a}$ & $\begin{array}{l}\text { Al ubicarnos en otro punto dentro de nuestro Sistema Solar podríamos } \\
\text { observar importantes cambios en las formas de las constelaciones que } \\
\text { conocemos }\end{array}$ & 76,1 \\
\hline $13 \mathrm{a}$ & Las estrellas más calientes tienen color rojo o naranjo & 52,1 \\
\hline $14 \mathrm{a}$ & La energía del Sol proviene del calor remanente del Big Bang & 34,3 \\
\hline $15 \mathrm{a}$ & $\begin{array}{l}\text { La luminosidad es inversamente proporcional a la distancia que hay } \\
\text { hasta la fuente que emite la luz }\end{array}$ & 72,9 \\
\hline $16 \mathrm{a}$ & $\begin{array}{l}\text { Las ondas de radio se propagan mucho más lentamente que la luz } \\
\text { visible }\end{array}$ & 73,2 \\
\hline $17 \mathrm{a}$ & $\begin{array}{l}\text { El calentamiento global es causado principalmente por la destrucción } \\
\text { de la capa de ozono }\end{array}$ & 54,9 \\
\hline
\end{tabular}


Estudios Pedagógicos XLVII N² 2: 247-268, 2021

CONCEPCIONES ALTERNATIVAS SOBRE ASTRONOMÍA EN ESTUDIANTES DE EDUCACIÓN BÁSICA Y MEDIA

DE LA REGIÓN METROPOLITANA DE CHILE

Tabla 10. Concepciones alternativas recurrentes en estudiantes de cuarto medio

\begin{tabular}{|c|c|c|}
\hline $\mathrm{N}^{\circ}$ & Concepción alternativa & $\%$ de respuestas \\
\hline $1 b$ & En el espacio no hay gravedad & 51,2 \\
\hline $2 b$ & La atmósfera aumenta el peso de los objetos & 34,1 \\
\hline $3 b$ & $\begin{array}{l}\text { La distancia Tierra-Luna es estimada como máximo en un } 10 \% \text { del } \\
\text { valor real }\end{array}$ & 48,8 \\
\hline $4 \mathrm{~b}$ & $\begin{array}{l}\text { La Estación espacial internacional se ubica aproximadamente a } \\
\text { media distancia entre la Tierra y la Luna }\end{array}$ & 39,0 \\
\hline $5 b$ & $\begin{array}{l}\text { La luminosidad de la Luna varía notoriamente durante una misma } \\
\text { noche }\end{array}$ & 53,7 \\
\hline $6 \mathrm{~b}$ & La luna se encuentra en fase llena cuando ocurre un eclipse solar & 65,0 \\
\hline $7 b$ & $\begin{array}{l}\text { Las estaciones del año se producen exclusivamente por la variación } \\
\text { de la distancia Tierra-Sol }\end{array}$ & 46,3 \\
\hline $8 \mathrm{~b}$ & El Sol se ubica en el cenit a lo menos un día en el año & 85,4 \\
\hline $9 b$ & El Sol se ubica en el cenit todos los días del año al mediodía & 51,2 \\
\hline $10 \mathrm{~b}$ & El Sol recorre distintas constelaciones en el cielo a lo largo de un día & 70,7 \\
\hline $11 b$ & $\begin{array}{l}\text { Al ubicarnos en otro punto dentro de nuestro Sistema Solar podríamos } \\
\text { observar importantes cambios en las formas de las constelaciones que } \\
\text { conocemos }\end{array}$ & 51,2 \\
\hline $12 b$ & Las estrellas más calientes tienen color rojo o naranjo & 43,9 \\
\hline $13 b$ & $\begin{array}{l}\text { La luminosidad es inversamente proporcional a la distancia que hay } \\
\text { hasta la fuente que emite la luz }\end{array}$ & 56,1 \\
\hline $14 b$ & $\begin{array}{l}\text { Las ondas de radio se propagan mucho más lentamente que la luz } \\
\text { visible }\end{array}$ & 51,2 \\
\hline
\end{tabular}

De las concepciones alternativas identificadas en estudiantes de octavo básico (Tabla 9) sólo tres de ellas (2a, 14a y 17a) no se repiten con una frecuencia considerable (menor al $30 \%$ ) en alumnos de cuarto medio, lo que da cuenta que la mayoría de ellas están arraigadas en las representaciones mentales de los estudiantes. Cabe mencionar que, gran parte de las concepciones alternativas presentes en ambos grupos, aparecen con una disminución en la frecuencia para los estudiantes de cuarto medio, sin embargo, están lejos de desaparecer en las respuestas, ya que su recurrencia sigue siendo considerable (entre el $34 \%$ y el $85 \%$ ).

Por otra parte, hay concepciones alternativas que se presentaron con mayor frecuencia en el grupo de cuarto medio que en el de octavo básico (7b, 8b y 9b), específicamente en los temas de "Estaciones del año" y "Movimientos del Sol en el cielo". La primera de ellas podría explicarse debido a que cuando se estudia la trayectoria elíptica que tiene la Tierra alrededor del Sol, suelen emplearse esquemas exagerados sobre su excentricidad, por tanto, es probable que los estudiantes piensen que la Tierra presenta notorias aproximaciones y 
alejamientos del Sol a lo largo de su trayectoria. Además, se puede estar afianzando aún más esta concepción alternativa debido a las experiencias cotidianas de que, cuando se está más cerca de una fuente de calor (por ejemplo, de una fogata encendida), se alcanza una mayor temperatura, que, si uno se sitúa lejos de ella. El segundo tema, referido a Movimientos del Sol en el cielo, deja en evidencia que los estudiantes realizan observaciones superficiales de los fenómenos del cielo que se producen todos los días, ya que es notorio, para una observación directa desde la latitud de Santiago de Chile, que el Sol no pasa por el cenit, y más si esta investigación se realizó en días de invierno, donde el Sol se ubica más próximo al horizonte durante toda una tarde. Sin duda, conocer con mayor profundidad sobre por qué estas concepciones alternativas se presentan más frecuentemente en estudiantes con mayor nivel educacional puede ser un tema interesante para la realización de futuras investigaciones.

Además, la información que se pudo recoger en los grupos focales, y que no se detallan en las tablas 9 y 10, permite reconocer que ambos grupos de estudiantes presentan concepciones alternativas sobre las distancias Tierra-Luna y Tierra-Marte, considerándolas, frecuentemente, mucho más pequeñas de lo que son realmente, lo que se podría extrapolar también a las dimensiones del Sistema Solar en general. Otro punto a considerar dentro de las concepciones alternativas reconocidas en los grupos focales es lo referido a la información que nos entrega el brillo de las estrellas: por un lado, los estudiantes de octavo básico reconocen que las estrellas más brillantes son más jóvenes, y a medida que pasan los años disminuyen su brillo, mientras que los estudiantes de cuarto medio afirman que las estrellas más brillantes se encuentran más cercanas a nosotros, en comparación a las de menor luminosidad.

Al analizar los resultados obtenidos en investigaciones previas (Camino, 1995; Atwood y Atwood, 1995; Atwood y Atwood, 1996; Parker y Heywood, 1998; Vega, 2001; Trumper, 2003; Martínez-Sebastià, 2004; Rutherford, 2004; Pedrochi y Neves, 2005; OganBekiroglu, 2007; Mulholland y Ginns, 2008; Varela et al., 2015; Huerta, 2017), se puede observar que, tanto los profesores en ejercicio como los profesores en formación, tienen concepciones alternativas muy similares a las que se han identificado en esta investigación, pese a la gran diferencia que existe tanto en edad como en nivel de educación de los sujetos. Ante esto, se hace necesario crear programas de formación para los tres grupos (estudiantes, profesores en formación y profesores en ejercicio), que permitan enfrentar estas dificultades; lo que supone un desafío para repensar tanto los cursos de astrofísica de pregrado como los programas de formación continua. Estos programas deben ayudar a transformar estas concepciones, y al mismo tiempo, desde la didáctica de la física, se hace evidente la necesidad de generar propuestas que posibiliten la construcción de estrategias para trabajar nociones y fenómenos de astrofísica en la educación básica y media. Incluso, para el caso de los profesores en formación y ejercicio, investigaciones sobre el Conocimiento Didáctico de los Contenidos (CDC) relacionados con Astronomía, permitiría reconocer sus concepciones, y al mismo tiempo, diseñar secuencias de aprendizaje que tengan impacto en las clases de física.

Los resultados de esta investigación representan una importante fuente de información para los docentes, ya que, como se ha argumentado anteriormente, considerando lo recurrente que son los esquemas mentales de los alumnos, las concepciones alternativas deben tenerse en cuenta para desarrollar estrategias de enseñanza que permitan explicitarlas, trabajarlas y mejorarlas. Esto implica una labor docente que mira la continuidad formativa de sus 
estudiantes, es decir, que pueda evaluar las concepciones de sus estudiantes en los distintos niveles, ya que, como se pudo apreciar en los resultados, muchas de ellas son persistentes y difíciles de superar tanto en alumnos de educación básica como en educación media.

Cabe mencionar que, como este estudio se aplicó sólo en la Región Metropolitana de Chile, para extrapolar sus resultados, se hace necesario el desarrollo de investigaciones con estudiantes de diversos tipos de establecimientos y ciudades a lo largo del país; de esa manera se podría alcanzar representatividad a nivel nacional, y, por tanto, generalizar los resultados.

\section{REFERENCIAS BIBLIOGRÁFICAS}

Atwood, V. \& Atwood, R. (1995). Preservice elementary teachers' conceptions of what causes night and day. School Science and Mathematics, 95(6), 290-294.

Atwood, R. \& Atwood, V. (1996). Preservice elementary teachers' conceptions of the causes of seasons. Journal of Research in Science Teaching, 33(5), 553-563.

Ausubel, D. P., Novak, J. D. \& Hanesian, H. (1983). Psicología educativa: un punto de vista cognoscitivo. Trillas.

Braun, V. \& Clarke, V. (2006). Using thematic analysis in psychology. Qualitative research in psychology, 3(2), 77-101.

Bretones, P. S. \& Compiani, M. (2011). Conceptual evolution of teachers about the daily motion of the celestial sphere. Ciência \& Educação (Bauru), 17(3), 735-755.

Cabero, A. J. \& Llorente, C. M. (2013). La aplicación del juicio de experto como técnica de evaluación de las tecnologías de la información y comunicación (TIC). Revista de Tecnología de Información y Comunicación en Educación, 7(2), 11-22.

Camino, N. (1995). Ideas previas y cambio conceptual en Astronomía. Un estudio con maestros de primaria sobre el día y la noche, las estaciones y las fases de la luna. Enseñanza de las ciencias, 13(1), 81-96.

Campanario, J. M. \& Otero, J. (2000). Más allá de las ideas previas como dificultades de aprendizaje: las pautas de pensamiento, las concepciones epistemológicas y las estrategias metacognitivas de los alumnos de Ciencias. Enseñanza de las ciencias: revista de investigación y experiencias didácticas, 18(2), 155-169.

Cubero, R. (1989). Cómo trabajar con las ideas de los alumnos. Díada.

(1994). Concepciones alternativas, preconceptos, errores conceptuales... ¿distinta terminología y un mismo significado? Revista investigación en la escuela, 23, 33-42.

Cuellar Z. (2009). Las concepciones alternativas de los estudiantes sobre la naturaleza de la materia. Revista Iberoamericana de Educación, 50(2), 1-10.

Dove, J. (2002). Does the man in the moon ever sleep? An analysis of student answers about simple astronomical events: a case study. International Journal of Science Education, 24(8), 823-834.

Driver, R. (1993). Una visión constructivista del aprendizaje y sus implicaciones para la enseñanza de las Ciencias. Diez años de investigación e innovación en enseñanza de las ciencias. Centro de publicaciones del Ministerio de Educación y Ciencia CIDE.

Driver, R., Guesne, E. y Tiberghien, A. (1989). Ideas científicas en la infancia y la adolescencia. Morata.

. (1999). Ideas científicas en la infancia y la adolescencia, 8. Morata.

Flick, U. (2004). Triangulation in qualitative research. A companion to qualitative research, 3, 178-183.

Gangui, A., Iglesias, M. \& Quinteros, C. (2007). Astronomía en la escuela: situación actual y perspectivas futuras. Actas de la XV Reunión Nacional de Educación en la Física, 68-80.

. (2010). Indagación llevada a cabo con docentes de primaria en formación sobre temas básicos de Astronomía. Revista Electrónica de Enseñanza de las Ciencias, 9(2), 467-486. 
Giordan, A. (1996). ¿Cómo ir más allá de los modelos constructivistas? La utilización didáctica de las concepciones de los estudiantes. Revista Investigación en la Escuela, 28, 7-22.

Hamui-Sutton, A. \& Varela-Ruiz, M. (2013). La técnica de grupos focales. Investigación en educación médica, 2(5), 55-60.

Hernández, R., Fernández, C. \& Baptista, P. (2014). Metodología de la investigación. Editorial Mc Graw Hill.

Huerta Cancino, L. (2017). Concepciones alternativas mayoritarias sobre Universo en profesores de Física en formación. Estudios pedagógicos (Valdivia), 43(2), 147-162.

Hufnagel, B., Slater, T., Deming, G., Adams, J., Adrian, R. L., Brick, C. \& Zeilik, M. (2000). Precourse results from the Astronomy Diagnostic Test. Publications of the Astronomical Society of Australia, 17(2), 152-155.

Hurtado, J. (2006). Investigación cualitativa: comprender y actuar. La Muralla.

Iachel, G., Langhi, R. \& Scalvi, R. M. F. (2008). Concepções alternativas de alunos do ensino médio sobre o fenômeno de formação das fases da Lua. Revista Latino-Americana de Educação em Astronomia, 5, 25-37.

Kikas, E. (2004). Teachers' conceptions and misconceptions concerning three natural phenomena. Journal of Research in Science Teaching: The Official Journal of the National Association for Research in Science Teaching, 41(5), 432-448.

Kriner, A. (2004). Las fases de la Luna, ¿Cómo y cuándo enseñarlas? Ciência \& Educação, 10(1), 111-120.

Langhi, R. (2011). Educação em Astronomia: da revisão bibliográfica sobre concepções alternativas à necessidade de uma ação nacional. Caderno Brasileiro de Ensino de Física, 28(2), 373-399.

Langhi, R. \& Nardi, R. (2009). Ensino da astronomia no Brasil: educação formal, informal, não formal e divulgação científica. Revista Brasileira de Ensino de Física, 31(4), 4402-4412.

Martín del Pozo, R., Arillo, M., Fernández, P., Galán, P., García, E., González, M., Juanas, A., Reyero, C. y San Martín, C. (2013). Las ideas "científicas" de los alumnos y alumnas de primaria: tareas, dibujos y textos. Morata.

Martínez-Sebastià, B. (2004). La enseñanza/aprendizaje del modelo Sol-Tierra: Análisis de la situación actual y propuesta de mejora para la formación de los futuros profesores de primaria. Revista LatinoAmericana Educação em Astronomía - RELEA, 1, 7-32.

MINEDUC. (2013). Ciencias Naturales, Programa de estudio Tercero Año Básico, Ministerio de Educación. Santiago. https://www.curriculumnacional.cl/614/articles-20716_programa.pdf . (2016a). Ciencias Naturales, Programa de estudio Primero medio, Ministerio de Educación. Santiago. https://www.curriculumnacional.cl/614/articles-34456_programa.pdf . (2016b). Ciencias Naturales, Programa de estudio Segundo medio, Ministerio de Educación. Santiago. https://www.curriculumnacional.cl/614/articles-34453_programa.pdf

Mora, C. \& Herrera, D. (2009). Una revisión sobre ideas previas del concepto de fuerza. LatinAmerican Journal of Physics Education, 3(1), 13.

Mulholland, J. \& Ginns, I. (2008). College MOON project Australia: Preservice teachers learning about the moon's phases. Research in Science Education, 38(3), 385-399.

Ogan-Bekiroglu, F. (2007). Effects of model-based teaching on pre-service physics teachers' conceptions of the moon, moon phases, and other lunar phenomena. International Journal of Science Education, 29(5), 555-593.

Parker, J. y Heywood, D. (1998). The earth and beyond: developing primary teachers' understanding of basic astronomical events. International Journal of Science Education, 20(5), 503-520.

Patton, M. (1987). How to use qualitative methods in evaluation. Sage Publications, the International Professional Publishers.

Pedrochi, F. \& Neves, M. (2005). Concepções astronômicas de estudantes no ensino superior. Revista Electrónica de Enseñanza de las Ciencias, 4(2), 1-9.

Rutherford, L. B. (2004). Exploring alternative conceptions of teachers and informal educators about selected astronomy concepts (Doctoral dissertation), University of Cincinnati, Ohio. 
Estudios Pedagógicos XLVII N² 2: 247-268, 2021

CONCEPCIONES ALTERNATIVAS SOBRE ASTRONOMÍA EN ESTUDIANTES DE EDUCACIÓN BÁSICA Y MEDIA

DE LA REGIÓN METROPOLITANA DE CHILE

Schoon, K. J. (1995). The origin and extent of alternative conceptions in the earth and space sciences: A survey of pre-service elementary teachers. Journal of elementary science education, 7(2), 27.

Solbes, J. \& Palomar, R. (2011). ¿Por qué resulta tan difícil la comprensión de la Astronomía a los estudiantes? Didáctica de las ciencias experimentales y sociales. 25, 187-211

Solbes, J., Carrascosa, J. \& Furió, C. (2006). Las ideas alternativas sobre conceptos científicos: tres décadas de investigación. Alambique: Didáctica de las ciencias experimentales, 48, 64-77.

Solbes, J., Montserrat, R. \& Más, C. F. (2007). Desinterés del alumnado hacia el aprendizaje de la ciencia: implicaciones en su enseñanza. Didáctica de las ciencias experimentales y sociales, 21, 91-117.

Stahly, L. L., Krockover, G. H. \& Shepardson, D. P. (1999). Third grade students' ideas about the lunar phases. Journal of Research in Science Teaching: The Official Journal of the National Association for Research in Science Teaching, 36(2), 159-177.

Suárez-Iñiguez, E. (2005). ¿Es realmente necesaria la hipótesis al inicio de la investigación? Estudios políticos (México), 8(5), 43-56.

Suárez, C. (2016). La Tierra y el Universo: Evolución de las ideas del alumnado tras el proceso de enseñanza-aprendizaje (Trabajo de fin de grado). Universidad de Sevilla, España.

Trumper, R. (2001). A cross-age study of junior high school students' conceptions of basic astronomy concepts. International Journal of science education, 23(11), 1111-1123.

. (2003). The need for change in elementary school teacher training-a cross-college age study of future teachers' conceptions of basic astronomy concepts. Teaching and Teacher Education, 19(3), 309-323.

Trundle, K. C., Atwood, R. K. \& Christopher, J. E. (2002). Preservice elementary teachers' conceptions of moon phases before and after instruction. Journal of Research in Science Teaching: The Official Journal of the National Association for Research in Science Teaching, 39(7), 633-658.

- (2007). Fourth-grade elementary students' conceptions of standards-based lunar concepts. International Journal of Science Education, 29(5), 595-616.

Varela-Losada, M. M., Pérez-Rodríguez, U., Álvarez-Lires, M. \& Arias-Correa, A. (2015). Concepciones alternativas sobre Astronomía de profesorado español en formación. Ciência \& Educação (Bauru), 21(4), 799-816.

Varela, M., Pérez, U., Serrallé, J. \& Arias, A. (2013). Evolución de las concepciones sobre Astronomía de profesorado en formación tras una intervención educativa con actividades de simulación. Enseñanza de las Ciencias, 31, 3612-3617.

Vega, A. (2001). Tenerife tiene seguro de sol (y de Luna): representaciones del profesorado de primaria acerca del día y de la noche, Enseñanza de las Ciencias, 19(1), 31-44.

Zeilik, M. (2003). Birth of the astronomy diagnostic test: Prototest evolution. Astronomy Education Review, 1(2), 46-52.

Zeilik, M., Schau, C. \& Mattern, N. (1998). Misconceptions and their change in university-level astronomy courses. The Physics Teacher, 36(2), 104-107. 